\title{
M-type barium hexaferrite synthesis and characterization for phase shifter applications
}

\author{
A. T. Wise, ${ }^{1, a)}$ J. Rocks, ${ }^{1}$ D. E. Laughlin, ${ }^{1}$ M. E. McHenry, ${ }^{1}$ S. D. Yoon, ${ }^{2}$ C. Vittoria, ${ }^{2}$ \\ and V. G. Harris ${ }^{2}$ \\ ${ }^{1}$ Carnegie Mellon University, Pittsburgh, Pennsylvania 15213, USA \\ ${ }^{2}$ Northeastern University, Boston, Massachusetts 02115, USA
}

(Presented 18 November 2010; received 5 October 2010; accepted 23 November 2010; published online 13 April 2011)

\begin{abstract}
M-type barium hexaferrite films have been grown by liquid phase epitaxy and examined by $\mathrm{x}$-ray diffraction, scanning electron microscopy, atomic force microscopy, and conventional and Lorentz-mode transmission electron microscopy (TEM). These films exhibit a diamond chevron shaped "brick wall" microstructure with $c$-axis oriented hexaferrite platelets. The films are oriented with their $c$ axes in-plane, and parallel to the M-plane sapphire substrate, and exhibit a $30^{\circ}$ rotation about the $c$ axis with respect to the substrate. Rocking curves showed (20-20) and (22-40) FWHM values of $1.09^{\circ}$ and $1.56^{\circ}$, respectively, for the thinner of two samples, and $0.31^{\circ}$ and $0.50^{\circ}$ for the thicker sample. The magnetic domain structures have been characterized by Lorentz-mode TEM and the domain walls were found to be pinned to small angle tilt boundaries. Using the measured rocking curve values, the effect of the overall crystalline misorientation on the dispersion of the magnetocrystalline anisotropy of the samples is estimated to be less than half a percent. () 2011 American Institute of Physics. [doi:10.1063/1.3559471]
\end{abstract}

Since M-type barium hexaferrite was originally examined in the late 1930s by Adelskold, ${ }^{1}$ and further studied by Gorter and Braun at Philips in the $1950 \mathrm{~s},{ }^{2,3}$ the unique properties associated with its anisotropic magnetic and crystalline structures have made the system of great interest to both scientists and engineers. ${ }^{4-6}$ These properties include high permeability, electrical resisitivity, and high ferromagnetic resonance (FMR) frequencies, with relatively narrow FMR linewidths. Additionally, the ability to grow hexaferrite films with the crystallographic $c$ axis aligned either in plane or perpendicular to the plane provide these materials with magnetic anisotropy that make them suitable for various applications. Phase shifters, delay lines, filters, and antenna applications can be fabricated from films with in-plane anisotropy, and isolator and circulator applications can be fabricated from films possessing perpendicular magnetic anisotropy. ${ }^{7}$ While these properties are important in determining hexaferrites' utility as microwave materials, they also have excellent radiation resistance and high power handling capacities that make them ideal for next-generation microwave devices, especially for use in extreme environments. ${ }^{8,9}$

The structure of the hexaferrites is unique in that all the observed structures, including $\mathrm{M}$ type, $\mathrm{Y}$ type, $\mathrm{Z}$ type and so on, are made up of common subunits that are present in different numbers and repeat sequences. ${ }^{4,10}$ All subunits are built upon a close packed lattice of oxygen with different metal ion arrangements. For example, in the case of M-type barium hexaferrite, the stacking sequence is $\mathrm{RSR} * \mathrm{~S} *$ in which $\mathrm{R}$ represents a rhombohedral subunit and $\mathrm{S}$ represents

\footnotetext{
a) Author to whom correspondence should be addressed. Electronic mail: adamwise@andrew.cmu.edu.
}

a spinel subunit. The asterisk $(*)$ represents a $180^{\circ}$ rotation of the subunit about the $c$ axis.

In growing epitaxial films, the lattice mismatch between the substrate and the film causes crystalline domains to form. ${ }^{11}$ This generally has deleterious effects on the properties of the film for use at high frequencies. ${ }^{12}$ As the magnetic anisotropy directly impacts the frequency and FMR linewidth of phase shifters, reducing the crystalline misalignment is critical for creating high-quality phase shifter materials for next-generation applications.

The hexaferrite films used in this study were synthesized at Northeastern University. There, BaM crystals were grown by liquid phase epitaxy on $\mathrm{BaM}$ seed layers. The seed layers were grown onto $m$-plane $(10-10)$ sapphire $\left(\mathrm{Al}_{2} \mathrm{O}_{3}\right)$ substrates using pulsed laser ablation deposition (PLD) technique. A total thickness of 0.2 um was deposited for seed layers onto the surface of the substrates. The substrate, with seed layer, was then immersed into an isothermal BaM flux melt. The controlled lowering of the melt temperature resulted in the nucleation and growth of the BaM crystal. ${ }^{13}$

These samples were first examined using an alternating gradient magnetometer (AGM) for measurements of hysteresis loops (i.e. $M$ vs $H$ ) along the in-plane and out-of-plane directions. The exact volume of the samples could not be determined due to the high degree of surface roughness which scanning electron microscopy (SEM) examination revealed. It is not obvious if the roughness is exaggerated from excess flux on the surface of the crystal from the melt.

For X-ray diffraction (XRD) analysis, a Panalytical X'Pert XRD system employing $\mathrm{Cu} K \alpha$ radiation and highresolution X-ray optics was used. $\omega-2 \theta$ scans were acquired, showing the alignment of the film relative to the substrate normal. Phi $(\varphi)$ scans were taken to explore if directional variants 
were present and to determine the alignment of the substrate with respect to the film. Finally, rocking curves were performed on two major peaks, (20-20) and (22-40), for each film sample.

In acquiring SEM images, the samples were sputtercoated with $2 \mathrm{~nm}$ of Pt to reduce surface charging. They were imaged in an FEI Phenom tabletop SEM. The scale was calibrated, allowing for a length measurement error of less than $5 \%$. The samples were imaged both top-down, to view the surface morphology, and cross sectionally to measure sample thickness.

Due to the brittle nature of the hexaferrite samples, transmission electron microscopy (TEM) samples were prepared by focused ion beam (FIB) instead of the traditional mechanical thinning. The FIB process was carried out by first delaminating pieces of the film, then mounting the delaminated sections on half of a copper glider sample holder. This was then placed in an FEI Nova 600 dual-beam FIB for thinning and polishing. Final polishing left the sample with a window less than $25 \mathrm{~nm}$ thick near the sample edge.

The initial TEM examination was carried out using an FEI Tecnai F20 electron microscope with an Orius SC600 CCD imaging system. The instrument was operated in Lorentz mode, with the objective lens turned off during the initial characterization, leaving the sample in a magnetic field-free region to image magnetic domain walls. Once a domain wall had been identified and imaged in Lorentz mode, the objective lens was turned on and the machine operated in conventional TEM mode for further characterization of the area surrounding the domain wall. The measurements of crystal misorientation on either side of the domain wall were carried out in a JEOL-2000EX microscope equipped with a CoolSnap CCD camera.

The XRD analysis revealed that the sample was single crystalline, with the $c$ axis aligned in the sample plane, parallel to the $\mathrm{c}$ axis of the sapphire substrate, and with a $30^{\circ}$ rotation about the $c$ axis, providing $[101-0]_{\text {sub }} \|[11-20]_{\text {film }}$, and $[0001]_{\text {sub }} \|[0001]_{\text {film. }}$. The rocking curves showed a significantly higher crystal quality in the thicker of the two M-type hexaferrite samples. Specifically, the thinner sample, $t \sim 18 \mu \mathrm{m}$, showed FWHM values measured from rocking curves of $1.56^{\circ}$ for the $(22-40)$ reflection and $1.09^{\circ}$ for $(20-20)$, while the thicker sample, $t \sim 50 \mathrm{um}$, showed FWHM values of $0.50^{\circ}$ for the (22-40) reflection and $0.31^{\circ}$ for $(20-20)$. The rocking curves for the two samples about the (20-20) peak are shown in Fig. 1(a).

In- and out-of-plane magnetic moment data were acquired with an AGM. The easy- and hard-axis loops for the $18 \mu \mathrm{m}$ sample are shown in Fig. 1(b). The easy axis was directed normal to the short axis of the plateletlike growth structures seen in Fig. 2(a), indicating the easy axis, known to be the (0002) axis in these materials, lies in the plane of the film. This surface indicates a growth mode where plates of the material with their short axes aligned with the $c$ axis of the film have grown out from the surface, a pattern indicating preferential growth along crystal directions normal to the $c$ axis.

In this growth pattern we find that the consistent easy growth direction is along the basal plane. In the crystal struc- ture of the hexaferrites, very few attachment sites are provided on the exposed basal plane for different atomic species during growth. This is in contrast to the planes normal to the basal plane, where a greater number of attachment sites for different species are available. The result is a variation in growth rates along the different directions, resulting in the plateletlike structures illustrated in Fig. 2(b). The platelets grow vertically out from the substrate surface and come together at small angle tilt boundaries. The resulting microstructure is reminiscent of the "brick wall" structure observed in anisotropic high temperature superconducting (HTSC) materials with large $c / a$ aspect ratios, ${ }^{14-17}$ but with the $c$ axis lying in the film plane. In HTSCs, misorientation of boundaries to which the $c$ axis is normal decreases the critical current density. In anisotropic hard ferrites both in- and out-of-plane misorientations increase anisotropy field dispersion and increase the FMR linewidth.

The Lorentz TEM analysis shows that few magnetic domain boundaries are present, fewer than the number of crystallographic domains. The sample examined in the TEM, as prepared by FIB, had an electron transparent region approximately $10 \mu \mathrm{m}$ deep and nearly $35 \mu \mathrm{m}$ across. Since the size of the platelets is significantly smaller than this, a number of subgrains were visible in the thin area of the sample. However, very few domain walls were observed. A domain wall image is seen in Fig. 3, which shows the Fresnel overfocus, and underfocus images. The domain wall is visible as the line that changes contrast going from overfocus to underfocus and vanishes in the focused image. Further examination of the domain wall location using selected area electron diffraction (SAED) indicated that the domain walls were pinned to small angle tilt boundaries. The boundary tilt measured in the case of the domain wall shown in Fig. 3 was approximately $1^{\circ}$. This tilt was measured with repeated measurements of diffraction pattern misorientation taken on either side of the domain wall. In situ, an abrupt change in the center of the circle of bright diffracted spots indicating the Ewald sphere was readily visible, though this did not translate well into the recorded images.

With these data on the crystal structure of the materials, we are able to make estimates of the effect that the measured amounts of crystalline imperfections will have on the performance of these films in device applications. Device performance is directly related to the crystal anisotropy, and crystalline misalignment such as that measured in these samples will decrease that anisotropy. Ohodnicki et al. ${ }^{10}$ provided the following equation in spherical coordinates for the crystalline anisotropy energy of a sample containing misoriented grains:

$$
E_{A}\left(\theta^{\prime}, \varphi^{\prime}\right)=\int_{\varphi=0}^{\varphi=2 \pi \theta=\pi / 2} \int_{\theta=0} \rho(\theta, \varphi) K_{\text {Ucrystal }} \sin ^{2}\left[\omega\left(\theta, \varphi, \theta^{\prime}, \varphi^{\prime}\right)\right] d \theta d \varphi,
$$

where $\rho(\theta, \varphi)$ is a function representing the probability of crystallites with easy axes lying between $\theta+d \theta$ and $\varphi+d \varphi$, and $\theta$ and $\theta^{\prime}$ are the angles between the substrate $c$ axis and $c$ axis of a particular grain, and the angle between the film magnetization direction and the $c$ axis of a particular grain, respectively. $\varphi$ and $\varphi^{\prime}$ are the corresponding values for 
a

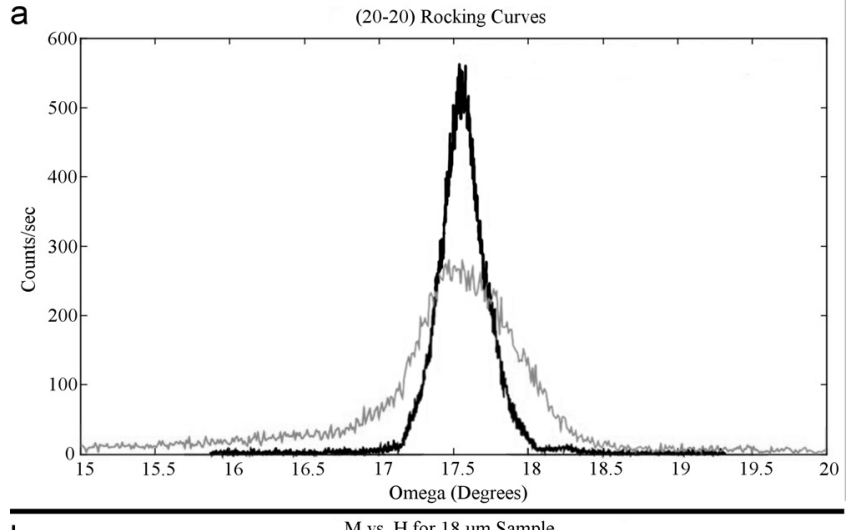

b

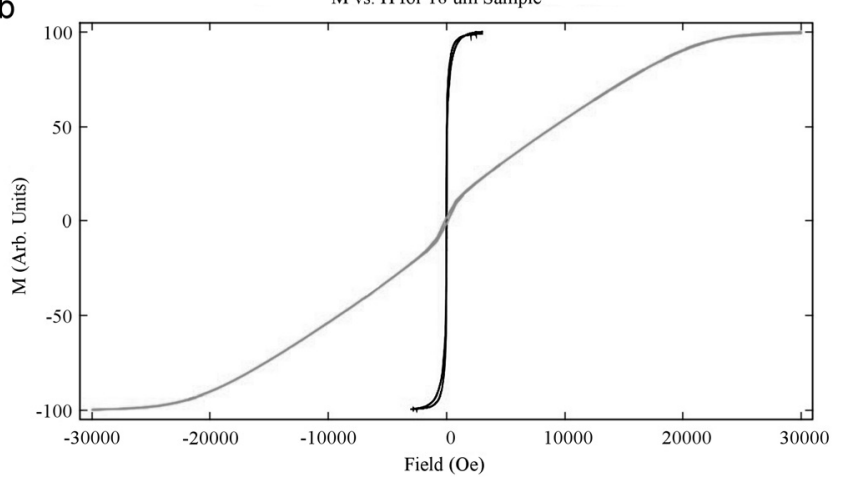

FIG. 1. (a) XRD rocking curves of (20-20) peak for (gray line) $18 \mu \mathrm{m}$ sample and (black line) $50 \mu \mathrm{m}$ sample. (b) $M$ vs $H$ loops for the $18 \mu \mathrm{m}$ sample. The black line was taken along the in-plane $c$ axis of the material and shows easy axis. The gray line was taken at $90^{\circ}$ to the $c$ axis and shows hard-axis behavior.

azimuthal angles, while $\omega\left(\theta, \varphi, \theta^{\prime}, \varphi^{\prime}\right)$ is the angle between the film magnetization defined by $\varphi^{\prime}$ and $\theta^{\prime}$ and the $c$ axes of grains defined by $\varphi$ and $\theta$. This is simplified by assuming a Gaussian distribution of the easy axes for $\rho$, giving:

$$
\rho(\theta, \varphi) \rightarrow \rho(\theta)=\rho_{0} e^{-\left(\theta / \theta_{0}\right)} \sin \theta,
$$

where $\rho_{0}$ is a normalization constant and $\theta_{0}$ is a measure of the average angular deviations of the $c$ axes of the film crystallites from the substrate $c$ axis. While the rocking curve data for the $(20-20)$ and $(22-40)$ reflections prove that this is not exactly true, we are able to find limiting values of the magnetocrystalline anisotropy deviation due to crystallite misorientation with this method.
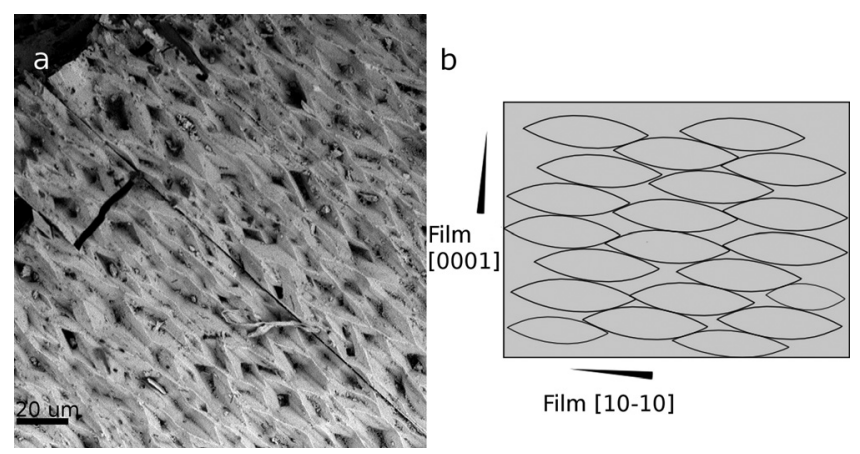

Film [10-10]

FIG. 2. (a) SEM of M-type barium hexaferrite film. (b) Schematic representation of platelet growth pattern in the hexaferrite films.

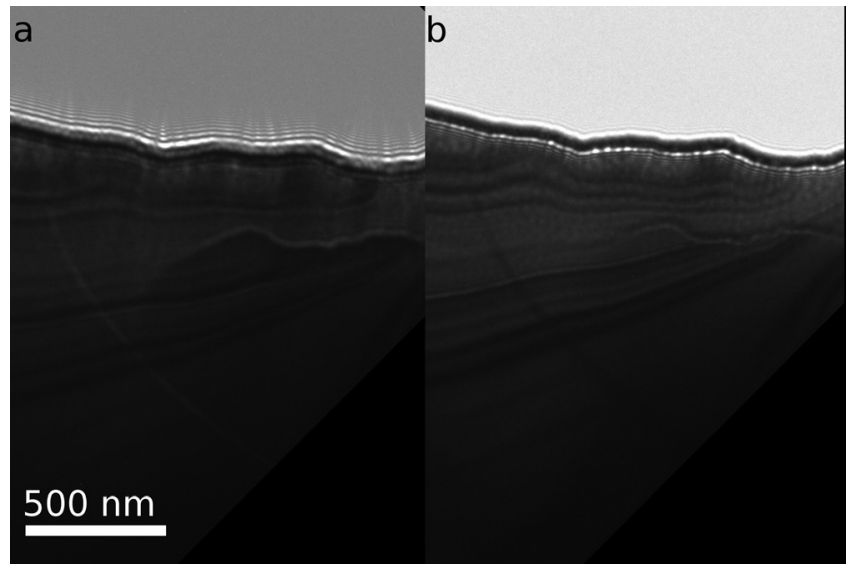

FIG. 3. (a) Underfocus Lorentz TEM image. (b) Overfocus Lortentz TEM image. The domain wall is visible as the line changing from bright to dark contrast in the two images.

By assuming a Gaussian distribution of crystalline misorientation in the rocking curves, we can set $\theta_{0}$ to $0.64^{\circ}$ for the thicker film and to $1.98^{\circ}$ for the thinner film, $3 \sigma$ in each case, leading to values of $K_{\text {Ueff }} / K_{\text {Ucrystal }}$ of $\sim 1$ and 0.995 , respectively. Therefore, the thicker film, with its better crystalline alignment shows nearly no reduction in the effective magnetocrystalline anisotropy, while the thinner film has a reduction of approximately half of one percent, still a reasonably small loss.

The authors would like to thank Jason Wolf and Tom Nuhfer of Carnegie Mellon University for their advice and counsel in regard to sample characterization, as well as the Earle and Mary Roberts Characterization Facility. This material is based upon work supported by the Naval Surface Warfare Center, Crane Division, United States of America under Contract No. N00164-09-C-GR34. Any opinions, findings and conclusions, or recommendations expressed in this material are those of the author(s) and do not necessarily reflect the views of the Naval Surface Warfare Center or Penn State EOC.

${ }^{1}$ V. Adelskold, Ark. Kemi, Mineral. Geol. 12A, 1 (1938).

${ }^{2}$ P. B. Braun, Philips Res. Rep. 12, 491 (1957).

${ }^{3}$ E. W. Gorter, Philips Res. Rep. 9, 295 (1954).

${ }^{4}$ J. Smit and H. P. J. Wijn, Ferrites: Physical Properties of Ferromagnetic Oxides in Relation to Their Technical Applications (Wiley, New York, 1959).

${ }^{5}$ J. A. Kohn, D. W. Eckart, and C. F. Cook, Jr., Science 172, 519 (1971).

${ }^{6}$ X. Obradors et al., J. Solid State Chem. 56, 171 (1985).

${ }^{7}$ X. Obradors et al., Mater. Res. Bull. 18, 1543 (1983).

${ }^{8}$ V. G. Harris et al., J. Magn. Magn. Mater. 231, 2035 (2009).

${ }^{9}$ Z. Cai et al., J. Phys. D 43, 095002 (2010).

${ }^{10}$ P. R. Ohodnicki et al., J. Appl. Phys. 103, 07 E514 (2008).

${ }^{11}$ P. R. Ohodnicki et al., Appl. Phys. 101, 09M521 (2007).

${ }^{12}$ D. Lisjak, D. Makovec, and M. J. Drofenik, J. Mater. Res. 19, 2462 (2004).

${ }^{13}$ S. G. Wang, S. D. Yoon, and C. Vittoria, J. Appl. Phys. 92, 6728 (2002).

${ }^{14}$ P. J. Kung et al., Physica C 249, 53 (1995).

${ }^{15}$ M. E. McHenry and R. A. Sutton, Prog. Mater. Sci. 38, 159 (1994).

${ }^{16}$ M. P. Maley et al., Phys. Rev. B 45, 7566 (1992).

${ }^{17}$ Z. H. Chen et al., Appl. Phys. Lett. 91, 182505 (2007). 